\title{
Forced Rayleigh scattering in mixtures of colloidal particles
}

T. Palberg, R. Simon, and P. Leiderer

Fakultät für Physik, Universität Konstanz, Konstanz, FRG
Konstanzer Online-Publikations-System (KOPS)

URL: http://www.ub.uni-konstanz.de/kops/volltexte/2007/3113/ URN: http://nbn-resolving.de/urn:nbn:de:bsz:352-opus-31134

\begin{abstract}
In this work, forced Rayleigh scattering is combined with a new deionization technique to measure the self-diffusion coefficient of strongly interacting charged polystyrene particles. For each measurement the, actually continuous, deionization process is stopped, allowing the suspension to relax from a sheared state to an equilibrium state. Conductivity and diffusion coefficient are monitored, both during this relaxation to equilibrium, and in dependence of the total deionization time. Relaxational behavior in the diffusion coefficient is observed in samples of both fluid and crystalline equilibrium state, while the conductivity remains unchanged. With increasing total deionization time the conductivity reaches a minimum and rises again to a stable plateau. The self-diffusion coefficient in equilibrium (i.e., at rest) also drops with increasing deionization time for monodisperse samples to unmeasurably low values. On the other hand, a finite, but small diffusion coefficient is found for the charge bidisperse sample.
\end{abstract}

Key words: Latex spheres; self-diffusion; forced Rayleigh scattering; crystallization; binary mixtures

\section{Introduction}

Strongly interacting colloidal suspensions show a full range of phase behavior. Glasslike [1,3] and complex crystalline structures [2] were observed as well as bcc and fcc phases [3]. This indicates that these suspensions may play an important role as model systems for atomic substances to clarify problems of phase transitions on a microscopic scale. Transport properties both in the vicinity of critical points and near a phase transition have recently gained considerable interest [4]. The latter include phase transitions in samples at rest as well as in samples subjected to external fields.

The self-diffusion coefficient [5] is a significant parameter to characterize phases and phase transitions. It can be measured reliably by the technique of forced Rayleigh scattering (FRS) [6], which was first applied to colloids by Chaikin and Dozier [7]. In contrast to other light-scattering techniques, FRS can especially be applied to mixtures of colloidal particles [8] as it can selectively detect one species alone. Here, we present first results on monodisperse and bidisperse latices obtained with a FRS setup and a new cyclic deionization method. The phase transition monitored is the crystallization from a supercooled melt respectively from a shear molten state.

\section{Experimental}

The particles used in this work are carboxylated polystyrene latices (Seradyn), referred to as C109, with a nominal diameter of $109 \mathrm{~nm}$ and a surface charge of $0.13 \mathrm{Cm}^{-2}$. The hydrodynamic diameter, determined by dynamic light scattering, is $r_{h}=$ $95 \mathrm{~nm}$, the effective surface charge determined by torsional resonance detection is $Z^{*}=540 \pm 20$, and the effectively transported charge from low-frequency conductivity and electrophoresis is $Z^{\mathrm{TR}}=$ $460 \pm 20$.

To obtain FRS active particles, a latex suspension of volume fraction $\Phi=5 \%$ was mixed with a solution of the spiropyrane dye "6 nitro-BIPS" (6nitro-1', 3', 3'-trimethyl-5' methoxyspiro-[2H-1-benzopyran-2,2'-indoline]) (Aldrich, FRG) in p-xylene $(99+\%$, Alrich, FRG). The emulsion was stirred 
d the xylene was removed by evaporation. The iydrodynamic diameter of dyed particles is $100 \mathrm{~nm}$, close to the original value, indicating almost complete removal of xylene. From conductivity measurements the charge is estimated to be about $40 \%$ of the charge of the undyed particles.

From both the dyed and undyed particles stock suspensions were prepared and stored over mixed bed ion exchange resin. These stock suspensions were further diluted with doubly distilled water (conductivity $55 \mathrm{nS} / \mathrm{cm}$ ) and glycerol (Rotipuran, Roth, FRG), filtered to remove dust and aggregates, and filled into a circulatory deionization set-up. Details on the set-up are given elsewhere [9]. Driven by a peristaltic pump (Ismatec, FRG) the suspension circulates through an exchange cell, a conductometer cell, and the FRS cuvette or any other experimental cell, all connected with Teflon and Tygon tubings (Reichelt Chemie, FRG). The exchange cell is filled with mixed bed ion exchange resin. Conductivity is measured with a conductometer and a platinic electrode (LF530 + LTA01, WTW, FRG). During the FRS measurements, made in a rectangular cell (Rank, Bottisham, UK), the tubings were blocked to prevent convection.

Care was taken that no coagulation at the ion-exchange resin took place and that the particle concentration remained constant. While pumping the suspension through the circuit the conductivity drops, goes through a minimum, and finally reaches a stable plateau. If at any time the process is stopped or the ion exchanger bypassed, the conductivity stays essentially at its value. A calibration of conductivity vs salt concentration can in principle allow measurements at known salt concentration, but was not done here. So we only present conductivity and diffusion data vs total deionization time in the result section.

The optical set-up is shown in Fig. 1. The beam of an argon laser $\left(\lambda_{w}=360 \mathrm{~nm}, P=1-100 \mathrm{~mW}\right.$ ) (Innova 90, Coherent, USA) is split and recombined at the sample within an angle $\theta_{w}$. There a sinusoidal grid-like interference pattern with wavevector $q$ is produced:

$$
q=\frac{2 \pi}{d}=\frac{4 \pi \sin \left(\theta_{u^{\prime}} / 2\right)}{\lambda_{u}} .
$$

The dye molecule has two states: In the first state it absorbs ultraviolet light, and having absorbed a photon it changes to an excited state, where it ab- sorbs red light and therefore appears dark blue. The excited state has a relaxation time $\tau_{d}$ of several minutes. The absorbance is proportional to the intensity of the incident UV-light. Therefore, an absorption grid of the same wavevector $q$ is formed that now can be detected by a He-Ne laser $\left(\lambda_{r}=\right.$ $633 \mathrm{~nm}, P=0.5 \mathrm{~mW}$ ) (Novette, Uniphase, FRG). The intensity of the Bragg diffracted red light is monitored with a photodiode.

Since the irradiation time has to be much shorter than the diffusion time, the argon UV-laser is chopped by a shutter (F-301, Nikon, Japan).

Consider a periodic modulation of the density $\rho_{T}$ of tagged particles within a suspension of constant particle concentration:

$$
\rho_{T}(x, t=0)=\rho_{T}^{0}+\rho_{T}^{1} \cos (q x+\varphi) .
$$

Diffusion leads to a decay of the modulation. In systems without interactions between the particles the self-diffusion coefficient depends on the macroscopic viscosity of the solvent and is given by the Stokes-Einstein relationship.

$$
D=\frac{k_{B} T}{6 \pi \eta r} .
$$

If there are electrostatic and hydrodynamic interactions between the particles, one has to distinguish between the collective diffusion coefficient and the self-diffusion coefficient [5]. If these interactions are not affected by the state of the dye (which is assumed here), the decay of the grid is governed only by self-diffusion of the labeled particles [10]

$$
\rho_{\Gamma}^{1}(t)=\rho_{r}^{1}(0) e^{-i q^{2} t} .
$$

Typically, two different backgrounds add to the diffraction signal, resulting in a photodetector output:

$$
I(t)=I_{0}\left(e^{-t / \tau}+b\right)^{2}+c
$$

Here $b$ is a coherent and $c$ an incoherent background. The scattered intensity decays, not only because of the diffusional washing-out of the grid, but also because of the intrinsic dye relaxation. This leads to a modification of the relaxation time which depends on $q$ and the intrinsic dye relaxation time $\tau_{d}$ according to: 




PM OSZILLOSCOPE C LE M
$L_{W}$ WRITING LASER

$L_{R}$ READING LASER

M MIRRORS

$S$ SHUTTER

FO FOCUSSING OPTICS

BS BEAM SPLITTER

C CELL

BT BEAM TRAP

LE LENS

PM PHOTOPULTIPLIER
Fig. 1 Optical setup

To achieve a good signal-to-noise ratio several shots $(5-20)$ must be added and averaged. This was done with an IBM-AT compatible PC supplied with a datarecord card (Advantech, Taiwan).

\section{Results}

We here present data on three different samples of dyed spheres C 109 in 15\% mixtures of glycerol in water.

Sample 1 has a volume fraction of $0.14 \%$, sample 2 of $0.3 \%$. The samples are fluidlike and crystalline ordered, respectively, at rest at the lowest salt concentration obtainable. The third sample is a $1: 1$ mixture of dyed and undyed particles with a volume fraction of $0.27 \%$. In the final state of preparation this sample shows tiny crystallites that seem to be immersed in a second, non-crystalline phase.

Sample 1: In Fig. 2 the conductivity and the diffusion coefficient are plotted vs deionization time for sample 1. For each FRS measurement the deionization process is interrupted and the originally sheared suspension is left to relax. In this sample it is not observed that $D$ changes with time after the shearing was stopped. With increasing total deionization time the conductivity drops from initial values of $20 \mu \mathrm{S} / \mathrm{cm}$ to $0.17 \mu S / \mathrm{cm}$ where it reaches a minimum and rises again to a stable plateau value of $0.28 \mu \mathrm{S} / \mathrm{cm}$, a phenomenon also described in [9]. The diffusion coefficient decreases steadily with total deionization time to about one-

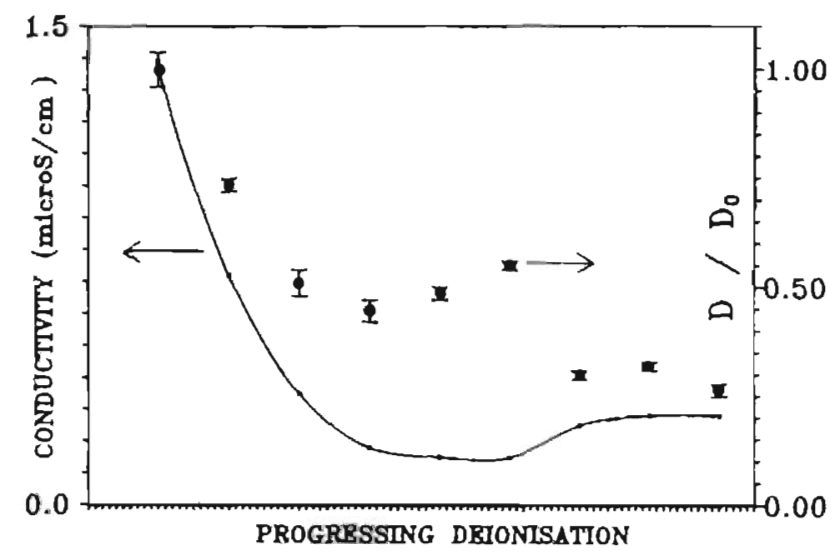

Fig. 2. Conductivity (solid line) and self-diffusion coefficient (circles) during the deionization progress in sample 1

third of the Stokes Einstein value $D_{0}$. At the end of the deionization process a pronounced DebyeScherrer ring is seen, indicating a fluid-like interparticle ordering induced by strong electrostatic interactions between the particles.

Sample 2: In the second sample with higher density the diffusion was measured in the same way. Note in Fig. 3 that the self-diffusion coefficient, measured 1 min after stopping the deionization process, differs from the value measured after leaving the suspension at rest for $10-30 \mathrm{~min}$. This is an indication that the suspension has not yet reached its equilibrium state at the time of the first measurement, but is still relaxing. If the suspension is highly deionized, the diffusion in the equilibrium state is below the sensitivity of the present setup. The time-dependence of the relaxation of the diffu- 


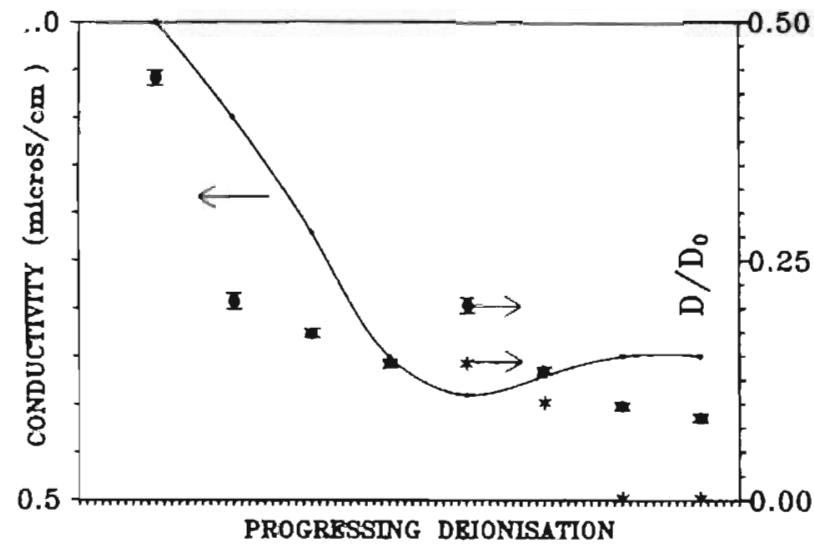

Fig. 3. Conductivity (solid line), self-diffusion coefficient immediately after stopping (circles) and self-diffusion coefficient some $10 \mathrm{~min}$ later (asterisks) during the deionization process in sample 2

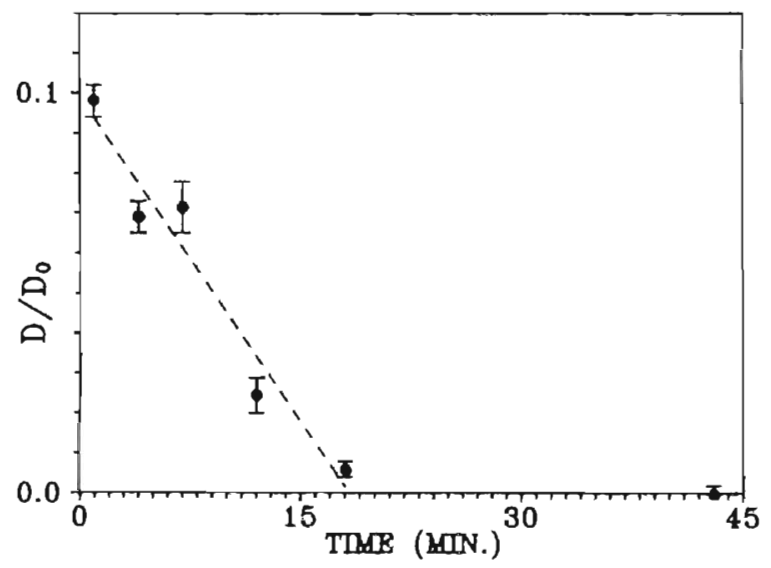

Fig. 4. Time evolution of the self-diffusion coefficient in sample 2

sion coefficient is drawn in Fig. 4. It is observed that $D$ drops approximately linearly with time. The initial state of the colloid may be compared with that of a supercooled liquid [10]. Since the thermal energy is low compared to the interaction energy the system tends towards a crystalline ordered state. In analogy to a supercooled liquid the liquid-like ordered colloid therefore crystallizes.

With increasing total deionization time the conductivity swings through a minimum and reaches a stable final value, similar to the behavior in the first sample. The diffusion coefficiont in the equilibrium state decreases steadily. In the fluid-like ordered phase the diffusion coefficient drops to about onefifth of the Stokes-Einstein value, and in the crystalline phase the coefficient is unmeasurably low. These data on equilibrium diffusion coefficients compare well with results from another group [7]. Light scattering produces a hexagonal pattern superimposed on a weak Debye-Scherrer ring, indicating that a bcc crystal with its (110) plane parallel to the cell wall was formed. There is no indication of phase separation in either monodisperse sample.

Sample 3: In the bidisperse sample 3 the diffusion of weaker charged particles was traced. Diffusion coefficients were again measured immediately after the deionization process was interrupted and in order to follow the development of the sample at some later time. In the liquid-like phase the diffusion coefficient changes with time after the deionization process is stopped. At the lowest salt concentration, we have no indication that the diffusion coefficient varies with time in contrast to the monodisperse sample.

In Fig. 5 the diffusion coefficient and the conductivity in the bidisperse sample are drawn is total deionization time. While the total deionization time increases, the conductivitity reaches a minimum and rises again, whereas the diffusion coefficient drops steadily to low values. At the end of deionization the liquidlike ordered state changes to a phase were tiny crystallites are seen. As mentioned before, these crystallites seem to be immersed in a fluid-like phase. For a polycrystalline solid, we would expect a much lower diffusion coefficient than we have measured. Unfortunately, we are not yet able to distinguish between decay mechanisms when two phases are present at the same time. Thus, the diffusion coefficient measured may be assigned to the fluid phase alone, but also may be a superposition of diffusion and translation of crystallites. Nevertheless, the data suggest the existence of phase separation in our system.

\section{Conclusions}

We have measured the diffusion coefficient during deionization of latex suspensions. The conductivity was monitored simultaneously. As deionization progresses the diffusion steadily slows down, whereas the conductivity always swings through a minimum before it reaches a constant value. This comparison indicates that the conductivity is not 




Fig. 5. Conductivity (solid line), self-diffusion coefficient immediately after stopping (circles) and self-diffusion coefficient some $10 \mathrm{~min}$ later (asterisks) during the deionization progress in sample 3

only dependent on the density of screening ions in the sample, but that the polystyrene spheres themselves contribute in a non-constant way to the conductivity.

In the strongly interacting colloid the self-diffusion coefficient relaxes at a timescale of minutes, which is by far longer than the time one particle needs to diffuse over typical interparticle distances.

In the bidisperse sample the diffusion coefficient does not drop to zero, but nevertheless reaches very low values. Relaxation phenomena after application of shear are also found for suspensions ordered fluidlike at rest. Some indications are found for a phase separation at very low salt concentrations. Further measurements will be required to answer the question if we mediate contributions from different phases to one diffusion coefficient or if the diffusion coefficient is slowed down homogeneously in the sample.
Acknouledgement

We gratefully acknowledge financial support from the Deutsche Forschungsgemeinschaft (SFB 306) and from ECIS.

\section{References}

1. Kesavamoorthy $R$ et al. (1988) J Phys Chem 21:4733

2. Hachisu $S$ et al. (1987) In: Safran S, Clark NA (eds) Physics of complex and supermolecular fluids. Wiley NY

3. Chaikin PM et al. (1989) Phys Rev Lett $62: 1524$

4. Bossis $G$ et al. (1990) Europhys Lett 11:133; Mimouni $Z$ et al. (1991) Prog Colloid Polym Sci 84; Hoffmann H, Kalus J et al. (1990) Prog Colloid Polym Sci 84

5. Hess W, Klein R (1983) Adv Phys 32:173; Nägele G et al. (1987) Prog Colloid Polym Sci 73:5

6. Pohl DW et al. (1973) Phys Rev Lett 31:32

7. Dozier WD, Thesis, UCLA (1986); Qui X et al. (1988) J Phys France 49:1043

8. Arauz-Lara JL et al. (1991) Prog Colloid Polym Sci 84; Aguanno B et al. (1991) Prog Colloid Polym Sci 84

9. Palberg $T$ et al. (1991) Prog Coll Polym Sci 84; Härtl W, Palberg T, Simnacher E, J Phys Chem to be published

10. Jäckle J (1986) Rep Prog Phys 49:171; Rössler E. (1990) Phys Rev Lett 65:1595

Authors' address:

R. Simon

Fakultät für Physik

Universität Konstanz

Postfach 5560

7750 Konstanz, FRG 